\title{
NOTE
}

\section{An improved video multiplexer for behavioral research}

\section{A. RODNEY WELLENS and DENIZ ERGENER University of Miami, Coral Gables, Florida}

Wellens and Revert (1979) described a solid-state circuit that allowed two sources of video information to be multiplexed and recorded using one videotape machine. On playback, the demultiplexed video signal allowed the original video channels to be viewed independently on separate television monitors. Recent changes in videocassette recorder technology have improved the playback quality of recorded images but disrupt the demultiplexing process when freeze frame or search options are selected. Improved multiplexing techniques that overcome these limitations are described.

Wellens and Revert (1979) described a time-division video multiplexer that allowed the simultaneous recording of two full-screen video images using a single videocassette recorder (VCR). The device has been used for recording facial expressions and eye movements of individuals conversing over two-way television (Wellens, 1979). A portable version of the device has been used in human factors research to provide a two-perspective record of elderly subjects performing household tasks (Faletti \& Clark, 1984). Similar technology has also been used in conjunction with special PLZT glasses to create stereoscopic television images (Bejczy \& Diner, 1985).

The heart of the original multiplexing system was an analog switch triggered by specially conditioned vertical sync pulses obtained from the input cameras' synchronizing source. The device alternated between video inputs at the video field rate of $1 / 60$ th of a second. The VCR thus recorded one set of images on odd video fields and the other set of images on even video fields. On playback, the interlaced images appeared to flicker between the two sources. When demultiplexed, the images could be separated and simultaneously displayed on independent monitors.

One useful feature of the multiplexing system was that two separate video images could be examined in detail while "freeze framing" the videotape. This was possible using VCRs available at the time of Wellens and Revert's (1979) article (e.g., Panasonic NV8200). At that time, most VCRs used the same set of playback heads

The authors' mailing address is: Department of Psychology, University of Miami, P.O. Box 248185, Coral Gables, FL 33124. both during freeze-framing and during normal playback. However, most VCRs that are now sold (e.g., Panasonic NV8500) use a special set of video heads for freeze and search operations. These new machines display only a single video field, rather than an interlaced video frame, during freeze operations and scan only odd video fields during slow motion and search operations. This change was engineered to eliminate "jitter" in picture playback due to small movements that may have occurred while recording between odd and even fields. Although this has improved instant replays of sporting events recorded in the normal way, the consequence for video multiplexing has been that only one of the two video sources recorded can be viewed during freeze, search, or slow motion operations using recently manufactured VCRs.

To overcome this restriction, the second author designed a faster multiplexing circuit that switched between video sources at the rate of 40 times per line (there are 525 lines of video in a standard video frame). Horizontal sync pulses were conditioned and used to trigger a high-speed oscillator that in turn drove an analog switch at a predetermined rate. This line-by-line multiplexing technique allowed sufficient information from both video sources to be available within each video field for demultiplexing on the newer VCRs. On playback, however, the new demultiplexed images gave a "screen door" or grating appearance due to the alternation of blank and filled video elements 40 times per line. This multiplexing scheme was improved by alternating the beginning video source for each line within a frame to create a "checkerboard" pattern. However, close examination of the display screen still resulted in an unacceptable level of "graininess." By experimenting with various switching rates, we soon discovered that slowing the multiplexing rate to 12 times per line resulted in a superior image. The current configuration now uses this slower switching rate combined with the checkerboard design to yield a multiplexed image of sufficient resolution to allow ratings of the same behavioral events for which the original field rate multiplexer was designed. A circuit diagram depicting the new multiplexing circuit is available from the authors at no charge.

\section{REFERENCES}

BejCzy, A., \& Diner, D. (1985). Human stereo vision research for space teleoperation. In NASA information sciences and human factors program annual report. 1985 (NASA technical memorandum 88380, pp. 24-25). Washington, DC: NASA Office of Aeronautics and Space Technology. 
FAlETti, M. V., \& ClaRK, C. (1984). A capability-demand approach to the aged in technological environments: A case study for improved task analysis. In P. K. Robinson, J. Livingston, \& J. Birren (Eds.), Aging and technological advances (NATO Conference Series, Vol. 24). New York: Plenum Press.
WelleNs, A. R. (1979). An interactive television laboratory for the study of social interaction. Joumal of Nonverbal Behavior, 4, 119-121. Wellens, A. R., \& REVERT, R. V. (1979). A time-division multiplexer-demultiplexer for duplex video recording and playback. $B e$ havior Research Methods \& Instrumentation, 11, 359-362.

(Manuscript accepted for publication August 17, 1986.) 